\title{
Identification morphométrique des populations de Tribolium castaneum Herbst (Coleoptera, Tenebrionidae) inféodées à trois céréales à Widou Thiengoli
}

\author{
Cheikh Abdou Khadre Mbacké DIA 1,2,3*, Adiouma Georges Robert Jacques SARR ${ }^{1,2,3}$, Ange KAFOM 1,2, \\ Toffène DIOME 1,2,3, Déthié Ngom 1,2, Cheikh THIAW 4, Saliou NDIAYE 5, Mbacké SEMBENE 1,2,3. \\ 1 Département de Biologie animale, Faculté des sciences et Techniques, Université cheikh Anta DIOP de Dakar. B.P. \\ 5005 Dakar, Sénégal. \\ ${ }^{2}$ Laboratoire d'Entomologie et d'Acarologie, Département de biologie Animale, Faculté des sciences et techniques, \\ Université Cheikh Anta DIOP de Dakar. B.P. 5005 Dakar, Sénégal. \\ ${ }^{3}$ Laboratoire de Biologie des Populations Animales Sahélo-Soudaniennes (BIOPASS). Institut de recherche pour le \\ développement (IRD) / Institut Sénégalais de Recherche Agricole (ISRA) Bel-Air B.P. 1386, Dakar, Sénégal. \\ ${ }^{4}$ CNRA de Bambey, Diourbel -PO 211, Sénégal \\ ${ }^{5}$ ENSA de Thiès, Thiès - N3, Sénégal \\ Auteur : Cheikh Abdou Khadre Mbacké DIA, email : cheikhabdoukhadrembacke.dia@ucad.edu.sn
}

Original submitted in on $27^{\text {th }}$ September 2017. Published online at www.m.elewa.org on $30^{\text {th }}$ November 2017 https://dx.doi.org/10.4314/jab.v119i1.9

\section{RÉSUMÉ.}

Objectif: Tribolium castaneum, est un coléoptère qui a la capacité d'infester toutes les céréales et légumineuses entreposées. Sa capacité à dérouler son cycle de développement sur divers substrats alimentaires a suscité des réflexions sur son adaptabilité morphologique. Cette étude a pour objectif d'identifier la morphologie de T. castaneum à Widou sur différentes céréales.

Méthodologie et résultats : Trois populations ont été définies en fonction de la céréale (maïs, mil ou riz) sur laquelle $T$. castaneum a déroulé son cycle de développement. Chacune des populations est constituée de 30 adultes mâles. Sur chaque adulte, 19 variables ont été mesurées. Les distances entre des yeux dorsalement et ventralement (Dyd, Dyv), la largeur du fémur_3 (If3) et des élytres (Ime) sont éliminées de l'analyse car ne présentant aucune variabilité entre les différents individus. Les 15 variables restantes sont utilisées pour des Analyses en Composante Principale. La taille est le premier facteur qui discrimine la population mil des autres. Suite à la transformation logarithmique des données brutes, l'Analyse discriminante a montré une différence de forme entre les trois populations. La classification ascendante hiérarchique a montré trois groupes morphométriques. Le groupe_1 dominé par les individus du mil est plus morphologiquement homogène. Le groupe_2 est dominé par les individus du riz et le groupe_3, le plus morphologiquement hétérogène, ne renferme que des individus du maïs.

Conclusion et application des résultats : Ce présent travail contribue à la mise en place d'une lutte intégrée contre $T$. castaneum. La connaissance de la diversité populationnelle de ce ravageur primaire des céréales stockées est nécessaire pour la mise en place une lutte adaptée selon sa variabilité morphogénétique.

Mots clés : Tribolium castaneum, biotype, ACP, morphométrie, AD, Widou Thiengoli, CAH. 
Dia et al., J. Appl. Biosci. 2017 Identification morphométrique des populations de Tribolium castaneum Herbst (Coleoptera, Tenebrionidae) inféodées à trois céréales à Widou Thiengoli

Morphometric identification of populations of Tribolium castaneum Herbst (Coleoptera, Tenebrionidae) subservient to three cereals at Widou Thiengoli

\section{ABSTRACT}

Objective: Tribolium castaneum is a beetle that has the ability to infest all stored cereals and legumes. Its ability to unfold its development cycle on various food substrates has prompted reflections on its morphological adaptability. This study aims to identify the morphology of T. castaneum in Widou Thiengoli on different cereals. Methodology and results: Three populations were defined according to cereals (maize, millet or rice) on which T. castaneum developed its development cycle. Each population consists of 30 adult males. On each adult, 19 variables were measured. The distances between the dorsal and ventral eyes (Dyd, Dyv), the width of the femur_3 (If3) and the elytra (Ime) are eliminated from the analysis because no variability between the different individuals. The remaining 15 variables are used for Principal Component Analyzes. Size is the primary factor that discriminates the millet population of others. Following the logarithmic transformation of the raw data, Discriminant Analysis showed a difference in shape between the three populations. Hierarchical ascending classification showed three morphometric groups. The group_1 dominated by biotype millet individuals is more morphologically homogeneous. Rice individuals dominate group 2 and group 3, the most morphologically heterogeneous, contains only maize individuals.

Conclusion and application of results : There is a morphological difference between the three populations of $T$. castaneum defined according to the food support, but the hypothesis of a morphological adaptation of $T$. castaneum on different food substrates can only be confirmed by varying the geographical sites. This present work contributes to the setting up of integrated struggle of $T$. castaneum. Knowledge of the population diversity of this primary pest of stored grains is necessary for the setting up of an adapted struggle according to its genetic variability.

Keywords: Tribolium castaneum, biotype, PCA, morphometry, AD, Widou Thiengoli, CAH.

\section{INTRODUCTION}

La biodiversité et la contribution à l'atténuation du changement climatique, à l'adaptation à ses effets et à l'amélioration de la sécurité alimentaire dans le Ferlo s'affichent parmi les objectifs majeurs du projet de la mise en place de la grande muraille verte dans le Sahel. Ancienne zone de transhumance et de sécurité des bergers peulhs, le Ferlo a subi d'importantes transformations qui défavorisent au fil du temps les pratiques culturales de ses terres. Ainsi, la quasi-totalité de la population pastorale de Widou Thiengoli s'abstient aux pratiques culturales de leurs terres et fait de l'élevage sa principale activité. De ce fait, le stockage des céréales importées d'autres localités est leur seul moyen d'approvisionnement alimentaire et est ainsi devenu une question de survie. Malgré ces méthodes déployées pour accéder à la sécurité alimentaire, cette dernière est loin d'être atteinte du fait des pertes post-récoltes qui ne cessent d'une part d'empêcher aux commerçants et producteurs de tirer pleinement profit de leurs produits commercialisés et d'autre part rendant difficile l'accès aux produits céréaliers sains. Ces pertes post-récoltes céréalières sont estimées, avant transformation, à 10-20\% des cultures stockées qui correspond à environ 4 milliards de dollars, représentant ainsi $13,5 \%$ de la valeur totale de la production céréalière des pays $\mathrm{du}$ Sahel (Ouédraogo et al., 2014). Ces pertes sont à $44 \%$ engendrées par les insectes (Sezonlin, 2006) dont Tribolium castaneum (Herbst) (Gueye et al., 2011) qui est reconnu comme étant le plus redoutable ravageur des céréales entreposées. Sachant que si les êtres vivants se transforment morphologiquement, c'est pour s'adapter aux changements de leur milieu, la capacité de $T$. castaneum à dérouler son cycle de développement sur différents supports alimentaires, a suscité des réflexions sur son adaptabilité morphologique face à ces différentes céréales qui lui offrent des ressources alimentaires et des conditions de survie optimales et différentes (Diome, 2014). D'où l'importance de la mise en place d'une lutte intégrée 
Dia et al., J. Appl. Biosci. 2017 Identification morphométrique des populations de Tribolium castaneum Herbst (Coleoptera, Tenebrionidae) inféodées à trois céréales à Widou Thiengoli

contre ce ravageur des denrées stockées afin de contribuer à la sécurité alimentaire au Ferlo dans le respect de l'environnement et de la santé publique. Pour cela, des connaissances préludes sur la morphologie et la génétique de $T$. castaneum sont nécessaires pour mieux appréhender une lutte intégrée contre ce ravageur. La capacité de $T$. castaneum à bien dérouler son cycle de développement sur différents supports alimentaires

\section{MÉTHODOLOGIE}

Échantillonnage et élevage de masse au laboratoire : l'échantillonnage a été réalisé durant le mois de mars à Widou Thiengoli sur du mil, du maïs et du riz stockés dans des magasins de commerce qui sont identifiés comme étant le seul moyen de stockage à Widou. Des quantités de chacune de ces céréales sont prélevées et mises dans des sachets en plastique. Ces derniers sont ramenés au laboratoire et les échantillons sont transférés dans des bocaux en verre à couvercles troués et recouverts de tissu moustiquaire, afin d'offrir un système d'aération à la population d'insectes contenue dans la spéculation et, en même temps, de créer une sorte de barrière pour éviter que les adultes émergeants quittent peut favoriser son adaptation aussi bien morphologique que génétique. Cette adaptation n'est que le résultat d'une forte diversité morphologie et/ou génétique entre populations trophiques. Cet article s'intéresse particulièrement à l'aspect morphologique de la diversité de $T$. castaneum. Pour cela, l'impact des différentes plantes hôtes sur la morphologie de T. castaneum sera étudié dans le but de tester son adaptation morphométrique trophique.

l'élevage après maturation. Les adultes qui émergent de cet élevage sont conservés dans des tubes $8 \mathrm{ml}$ contenant de l'alcool $96 \%$. Les adultes choisis sont distingués puis codés selon deux critères : l'insecte étudié ( $T$. castaneum) et la spéculation (mil, maïs et riz). Pour coder nos échantillons, nous avons utilisé la première lettre du nom de genre en majuscule et la première lettre du nom de l'espèce en minuscule (Tc), la première et la dernière lettre de la spéculation et enfin l'origine géographique (Tableau 1). Pour cette étude, 30 adultes sont récupérés dans chaque spéculation après élevage de masse.

Tableau 1: Nombre d'individus, leur spéculation et leur position géographique

\begin{tabular}{c|c|c|c|c|c}
\hline Localité & Plante hôte & Zone d'étude & Données géo -référencées & $\begin{array}{c}\text { Code de } \\
\text { l'insecte }\end{array}$ & $\begin{array}{c}\text { Nombre } \\
\text { d'Individus }\end{array}$ \\
\hline $\begin{array}{c}\text { Widou } \\
\text { Thiengoli }\end{array}$ & Mil & Sylvopastorale & $1^{\circ} 21^{\circ}$ Nord $15^{\circ} 29^{\prime}$ Ouest & TcmlWi & 30 \\
\cline { 2 - 4 } & Maïs & & & TcmsWi & 30 \\
\cline { 2 - 4 } & Riz & & & TcrzWi & 30 \\
\hline
\end{tabular}

Étude morphométrique : le matériel biologique utilisé pour la morphométrie est constitué de mâles de $T$. castaneum qui ont émergé après élevage de masse sur du mil, du maïs et du riz. Les insectes mâles qui ont émergé de l'élevage de masse sont utilisés pour la morphométrie. Seuls les mâles sont utilisés. L'insecte est placé à l'aide d'aiguilles sur le papier millimétré placé dans le champ de vision de la loupe allumée. L'insecte est disséqué en séparant d'abord ses trois parties à savoir la tête, le thorax et l'abdomen. Puis chaque partie est disséquée à son tour en séparant les différents articles qui le constituent. Les articles sont ensuite mesurés à l'aide du papier millimétré. Dix-neuf (19) articles et paramètres ont été mesurés sur chaque individus (Tableau 2). 
Dia et al., J. Appl. Biosci. 2017 Identification morphométrique des populations de Tribolium castaneum Herbst (Coleoptera, Tenebrionidae) inféodées à trois céréales à Widou Thiengoli

Tableau 2 : Différents articles mesurés, regroupés en fonction de la partie concernée de l'insecte

\begin{tabular}{l|l}
\hline Parties & Articles mesurés \\
\hline \multirow{3}{*}{ Tête } & $\begin{array}{l}\text { Longueur de l'antenne }(L m A) \text {, Distance minimale entre les yeux dorsalement }(D y d) \text {, Distance } \\
\text { minimale entre les yeux ventralement }(D y v), \text { Longueur maximale de la tête }(L m t) \text {, Largeur maximale } \\
\text { de la tête }(I m t)\end{array}$ \\
\cline { 2 - 3 } Thorax & $\begin{array}{l}\text { Longueur du pronotum au centre }(L p), \text { Largeur du pronotum }(I p) \text {, Distance entre le pronotum et le } \\
\text { thorax }(D p t), \text { Longueur du fémur 3 }\left(L f_{3}\right), \text { Largeur du fémur 3 }\left(I f_{3}\right), \text { Longueur du tibia 3 }\left(L t_{3}\right), \text {, Longueur } \\
\text { du Coxa 3 }\left(L C_{3}\right), \text { Longueur du tarse 3 }\left(L t a_{3}\right), \text { Longueur maximale de l'élytre }(L m e ́), \text { Largeur maximale } \\
\text { de l'élytre }(I m e ́)\end{array}$ \\
\cline { 2 - 3 } Abdomen & $\begin{array}{l}\text { Longueur maximale du premier sternite abdominal }(L / S), \text { Largeur maximale du premier sternite } \\
\text { abdominal }\left(I_{1} s\right), \text { Longueur maximale du pygidium }(L p y), \text { Largeur maximale du pygidium }(I p y) .\end{array}$ \\
\hline
\end{tabular}

Analyses statistiques

Choix du nombre $Q$ d'axes factoriels - $R$ version 3.2.3 (Bloomfield, 2014): le nombre Q d'axes factoriels adéquat pour la réalisation de l'analyse en composantes principales (ACP) est obtenu selon le critère du Coude qui permet d'obtenir le maximum d'inertie conservé avec le maximum de facteurs. Sur l'éboulis des valeurs propres, un décrochement est observé suivi d'une décroissance régulière. Les axes avant le décrochement représenteront le nombre d'axes factoriels à utiliser pour les ACP.

$A C P$, cercle de corrélation et contributions des variables - $R$ version 3.2.3 (Bloomfield, 2014) : en premier lieu, une analyse en composantes principales avec les données brutes est réalisée dans le but de voir le degré de corrélation entre les variables, avec le regroupement de celles-ci dans un seul plan d'une dimension de l'ACP. Une telle disposition des variables révèle la présence de l'effet taille. Suite à la transformation logarithmique des données, une autre ACP est générée afin de déterminer les composantes principales $(C P)^{i}$ pour chaque dimension. Ces dernières sont utilisées pour réaliser les analyses discriminantes (AD).

$A D$, discrimination des populations à priori $-R$ version 3.2.3 (Bloomfield, 2014) : une analyse discriminante (AD) des données brutes a été réalisée avec le logiciel afin de voir les différences de taille qui existent entre les biotypesii. Étant donné le degré de corrélation entre les variables avec les mensurations brutes, la répartition des individus observée n'est due qu'à des différences phénotypiques directement liées aux tailles moyennes des biotypes de $T$. castaneum. Pour cette raison, une autre $A D$ a été réalisée sous $R$ avec les $C P$ retenues des log-variables transformées afin de mettre en exergue les différences de forme entre les biotypes (groupes individus ayant déroulé leur cycle de développement sur un type de céréale).
Effet taille et transformation logarithmique des données (Darrroch \& Mosimann, 1985) : l'effet taille se manifeste par un cercle de corrélation qui regroupe toutes les variables dans un seul plan pour un axe donné. Le principe d'élimination s'agit d'une transformation logarithmique qui consiste à remettre tous les individus à la même taille, afin de n'observer sur l'ACP que des différences de forme (Darrroch \& Mosimann, 1985). La diminution du poids Taille se traduit par une baisse de la discrimination globale entre les populations et la réduction de la distance entre les barycentres des différentes populations. Cette transformation a été réalisée sous Excel version 2016.

Kruskal-Wallis \& Wilcoxon avec ajustement de Bonferroni - R version 3.2.3 (Bloomfield, 2014) Le test de Shapiro-Wilk est réalisé au préalable afin de tester sur chaque log-transformée si l'échantillon suit une loi normale ou non. Avec des P-valeurs hautement significatives (P-valeur $\ll<0,001)$, toutes les logtransformées ont montré que l'échantillon ne suit pas une loi normale. Tout compte fait, le test de Kruskal-Wallis (non paramétrique) est réalisé pour une comparaison multiple des moyennes des log-variables transformées entre biotypes. Le test de Wilcoxon avec l'ajustement de Bonferroni (non paramétrique) est réalisé pour tester la significativité des écarts de moyennes des logtransformées entre les biotypes pris deux à deux. Le seuil de significativité $(\alpha)$ est fixé à 0,05 .

CAH - R version 3.2.3 (Bloomfield, 2014) : les logvariables transformées qui ont des écarts de moyennes non significatifs entre les trois biotypes sont éliminés de l'analyse. En premier lieu, le coefficient d'agglomération est calculé afin de voir l'état de structuration $^{\text {iii }}$ de la population totale. Le nombre de classes du dendrogramme est ensuite déterminé par les graphiques des pertes relatives et des sauts d'inertie en fonction du nombre de classes. Le dendrogramme est 
Dia et al., J. Appl. Biosci. 2017 Identification morphométrique des populations de Tribolium castaneum Herbst (Coleoptera, Tenebrionidae) inféodées à trois céréales à Widou Thiengoli

tracé selon le nombre de classes retenues. L'indice moyen de Silhouette (IS) est calculé pour chaque groupe défini par la $\mathrm{CAH}$. Le graphique de Silhouette

\section{RÉSULTATS ET DISCUSSION}

Choix du nombre $Q$ d'axes factoriels: avec des mensurations identiques chez tous les individus, les variables telles que la distance entre les yeux dorsalement et ventralement (Dyd, Dyv), la largeur maximale des élytres (Ime) et la largeur du fémur de la troisième paire de pattes $\left(I f_{3}\right)$ sont éliminées de l'analyse. permet d'identifier les individus mal regroupés afin de retrouver le groupe voisin au sein duquel ils devaient appartenir.

L'éboulis des valeurs propres a révélé le décrochement après les deux premiers axes suivi d'une décroissance régulière (Figure 1). Donc, les deux premiers axes (le premier plan factoriel) sont retenus pour les analyses en composantes principales (ACP).

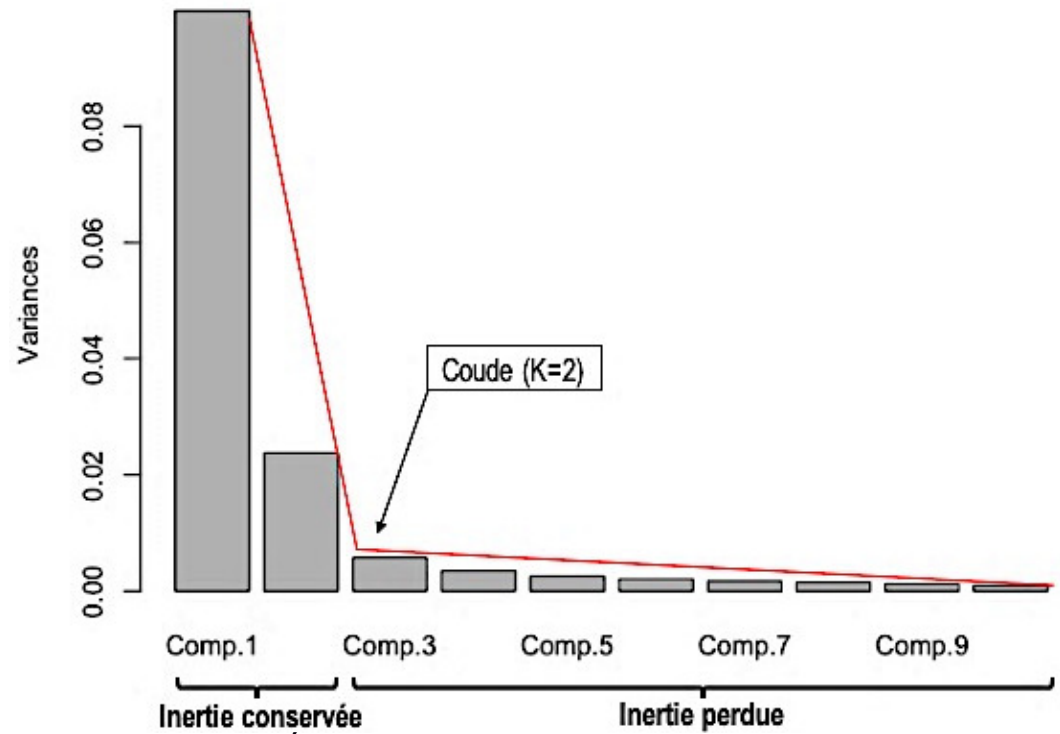

Figure 1 : Éboulis des valeurs propres du critère du Coude

\section{ACP et détermination des composantes principales :} 15 variables sont utilisées pour les analyses en composantes principales. L'ACP des données brutes montre que les deux premières dimensions expliquent $44,3 \%$ de la variabilité morphométrique totale (Figure $2 \mathrm{~A}$ ). Le regroupement des variables dans un seul plan suivant Dim_1 révèle la présence de l'effet taille. Parmi les $C P$, celles qui contribuent le mieux à la construction des deux axes (en rouge sur l'ACP) sont retenues. Parmi ces dernières, certaines d'entre-elles sont liéesiv à Dim_1 (Dpt (Cont_1 $=13,13)$, Lpy (Cont_1 $=15,46)$, Lta $_{3}=\left(\right.$ CoNT_$_{-} 1=$ 10,59)), d'autres à Dim_2 (Ip (Cont_2 = 17,69), L $L C_{3}$ $($ Cont_2 $=20,03)$ (Figure 2A). Bien que l'inertie totale de
l'ACP ait diminuée $(40,8 \%)$ suite à l'élimination de l'effet taille, la contribution des $\mathrm{CP}$ brutes qui ont été retenues a augmenté et elles restent les plus contributives à la construction des dimensions (Figure 2B). Les CP retenues (en rouge sur l'ACP), représentent les variables de forme ${ }^{v}$ (Figure 2B). Parmi ces dernières, Dpt (CONT_1 $=13,13)$, Lpy (Cont_1 $=15,46)$, Lta $_{3}=\left(\right.$ C ONT_1 $\left._{-}=10,59\right)$ restent toujours liées à Dim_1 qui a perdu $5,4 \%$ de son inertie, I1s (Cont_2 $=15,09)$, Ip (Cont_2 $=17,80)$ sont liées à Dim_2 qui a gagné $1,9 \%$ d'inertie et $L C_{3}$ (CONT_ $1=$ 6,95 Cont_2 $=8,88$ ) est liée à la fois aux deux dimensions (Figure $2 \mathrm{~B}$ ). 

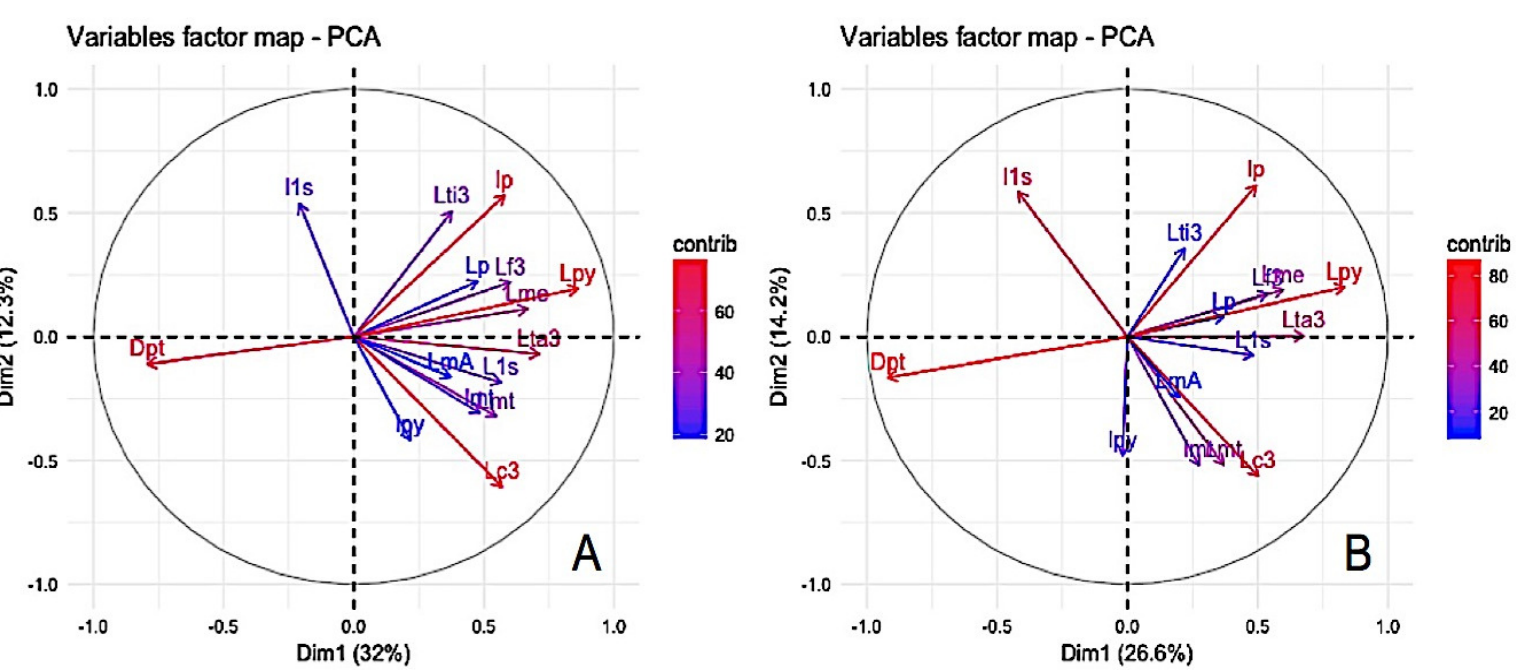

Figure 2 : ACP des données brutes (A) et des log-variables transforment (B) : la couleur de la variable détermine le niveau de significativité de sa contribution :

de bleu (non significatif) à rouge (significatif)
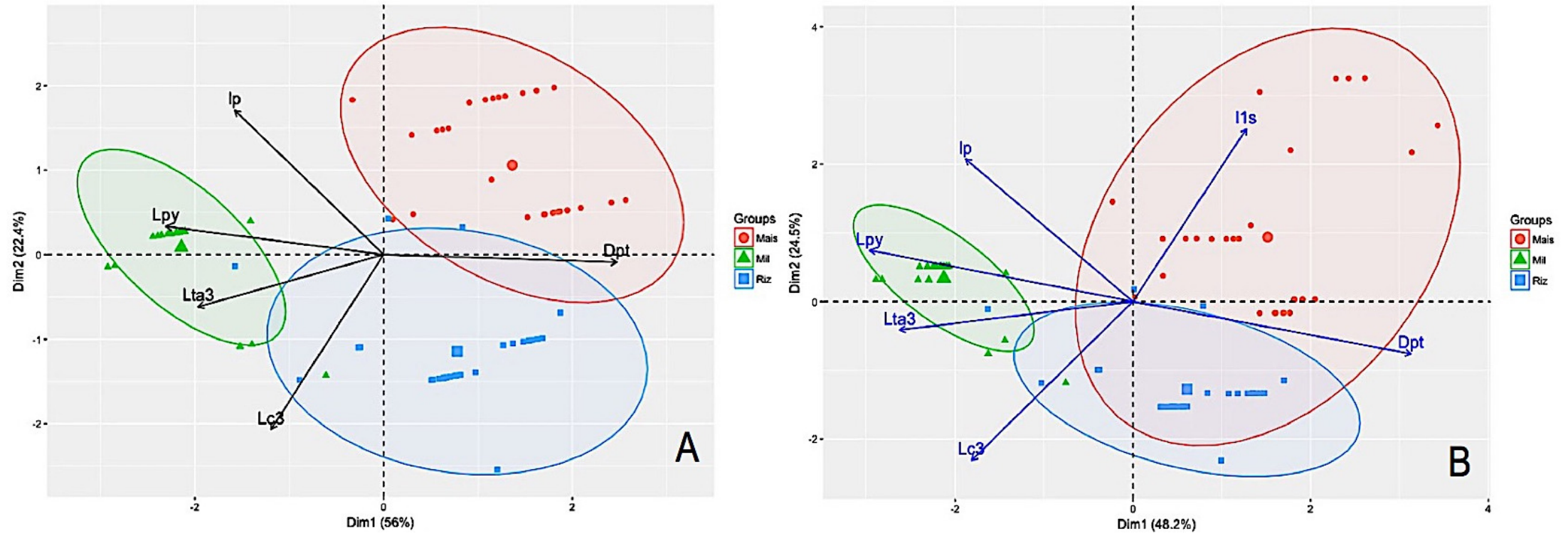

Figure 3 : AD des biotypes de Tribolium castaneum à Widou Thiengoli avec les données brutes (A) et les log-variables transformées (B) 

Herbst (Coleoptera, Tenebrionidae) inféodées à trois céréales à Widou Thiengoli

Analyse discriminante (AD) : La première dimension (Dim_1) de l'ACP, avec un pourcentage d'inertie de $41,1 \%$ du pouvoir discriminant total, a révélé une différence de taille entre le biotype mil et les autres. Le biotype mil, en plus d'avoir une faible dimension de la distance entre le pronotum et le thorax (Dpt), a les plus grandes dimensions de toutes les autres variables par rapport aux autres biotypes (Figure 3A). IL ressort de l'AD que le facteur taille intervient certes dans la différenciation entre biotypes mais l'analyse des logvariables transformées montre qu'il est loin d'être le seul. La Dim_1 oppose le biotype mil des autres par la distance entre le pronotum et le thorax (Dpt), la largeur du pronotum (IP), la longueur du pygidium (Lpy) et la longueur du tarse et du coxa de la troisième paire de pattes $\left(L t a_{3}, L c_{3}\right)$. La Dim_2 discrimine le biotype riz du biotype maïs par la largeur du ${ }^{\text {er }}$ sternite abdominal $\left(l_{1} s\right)$ et la longueur du coxa de la troisième paire de pattes $\left(L C_{3}\right)$ (Figure 3B).

Test de Tuckey-Kramer et particularité morphologique de chaque biotype : Seules les largeurs du 1er sternite abdominal $\left(I_{1} S\right)$ et la longueur du Coxa_3 $\left(L C_{3}\right)$ ont des écarts de moyennes significatifs entre les trois biotypes pris deux à deux. (Tableau 3). Les biotypes mil et maïs trouvent leur dissimilarité au niveau de la longueur du tibia, du tarse, du fémur et du coxa de la $3^{\text {ème }}$ paire de pattes $\left(L t i_{3}, L t_{3}, L c_{3}\right)$, de la longueur maximale des élytres $(L m e)$, de la longueur de l'abdomen ( $L p y$ et $L_{1} s$ ), de la longueur maximale des élytres (Lmé) et de la distance entre le pronotum et le thorax (Dpt) (Tableau 3). La dissimilarité morphologique entre les biotypes mil et riz s'identifie au niveau de la distance entre le pronotum et la tête $(D p t)$, de la longueur du 1 er sternite abdominal $\left(L_{1} s\right)$, de la longueur du coxa du fémur et du tibia de la 3ème paire de pattes $\left(L f_{3}, L C_{3}, L t i_{3}\right)$, de la longueur maximale des élytres (Lmé), de la largeur du pronotum (IP) et du pygidium ( $L p y, I p y)$ et la largeur du 1 er sternite $(/ 1 s)$. Étant plus morphologiquement proches, les biotypes maïs et riz trouvent leur dissimilarité au niveau de la largeur du 1er sternite abdominal $\left(I_{1} s\right)$, de la longueur du coxa de la 3ème paire de pattes $\left(L C_{3}\right)$ et de la largeur du pronotum (Ip) (Tableau 3).

Tableau 3 : Moyennes ( \pm Écart-type) log-variables transformées pour chaque biotype et test se significativité des écarts de moyennes.

\begin{tabular}{|c|c|c|c|c|}
\hline & Maïs & Mil & Riz & P-value (K-W) \\
\hline$D p t$ & $0.37 \pm 0.02^{\mathrm{a}}$ & $0.26 \pm 0.01^{b}$ & $0.36 \pm 0.03^{a}$ & *** \\
\hline$I_{1} \mathrm{~S}$ & $1.49 \pm 0.49 a$ & $1.24 \pm 0.04^{b}$ & $1.18 \pm 0.03^{c}$ & *** \\
\hline$L_{1 S}$ & $0.68 \pm 0.02^{a}$ & $0.75 \pm 0.03^{b}$ & $0.72 \pm 0.07^{\mathrm{ba}}$ & $* * *$ \\
\hline$L c_{3}$ & $0.57 \pm 0.01^{a}$ & $0.66 \pm 0.01^{b}$ & $0.67 \pm 0.02^{c}$ & *** \\
\hline$L f_{3}$ & $0.91 \pm 0.05 a b$ & $0.96 \pm 0.02^{a}$ & $0.75 \pm 0.05^{b}$ & ** \\
\hline $\operatorname{LmA}$ & $0.87 \pm 0.01^{a}$ & $0.89 \pm 0.04^{a}$ & $0.89 \pm 0.03^{a}$ & NS \\
\hline Lme & $2.41 \pm 0.11^{a}$ & $2.54 \pm 0.15^{b}$ & $2.42 \pm 0.08^{a}$ & ** \\
\hline Imt & $0.95 \pm 0.04^{a}$ & $0.96 \pm 0.01^{a}$ & $0.95 \pm 0.04^{a}$ & NS \\
\hline Lmt & $1.04 \pm 0.04^{a}$ & $1.06 \pm 0.02^{\mathrm{a}}$ & $1.05 \pm 0.03^{a}$ & NS \\
\hline Ip & $1.21 \pm 0.05^{\mathrm{a}}$ & $1.25 \pm 0.03^{a}$ & $1.18 \pm 0.03^{b}$ & $* * *$ \\
\hline$L p$ & $1.04 \pm 0.05^{a}$ & $1.06 \pm 0.01^{a}$ & $1.04 \pm 0.05^{a}$ & NS \\
\hline Ipy & $0.96 \pm 0.04 a b$ & $0.96 \pm 0.01^{a}$ & $0.97 \pm 0.01^{b}$ & $* * *$ \\
\hline Lpy & $0.48 \pm 0.02^{\mathrm{a}}$ & $0.56 \pm 0.02^{b}$ & $0.47 \pm 0.01^{a}$ & $* * *$ \\
\hline $\mathrm{Lta}_{3}$ & $0.59 \pm 0.04^{a}$ & $0.67 \pm 0.02^{b}$ & $0.62 \pm 0.05^{a b}$ & $\star * *$ \\
\hline $\mathrm{Lti}_{3}$ & $0.90 \pm 0.04^{b}$ & $0.96 \pm 0.01^{a}$ & $0.90 \pm 0.04^{b}$ & *** \\
\hline
\end{tabular}

Les lettes $a, b, c$ désignent des écarts de moyennes significatifs entre bio types à $\alpha=0,05$

" * " Significatif ; " ** " très significatif ; " *** " hautement significatif ; " NS " non significatif

Classification ascendante hiérarchique (CAH)

- Coefficient d'agglomération (AC): le coefficient d'agglomération est proche de 1 ( $A C=0,927$ ). II ressort de cette valeur que les individus de la population totale sont fortement structurés en plusieurs groupes.

- Détermination du nombre de classes retenues: le graphique de l'inertie relative en fonction du nombre de classes (Figure 4A) a révélé des découpages de la population globale en deux (point noir) et trois classes (point gris) comme étant les meilleures partitions de la $\mathrm{CAH}$. 

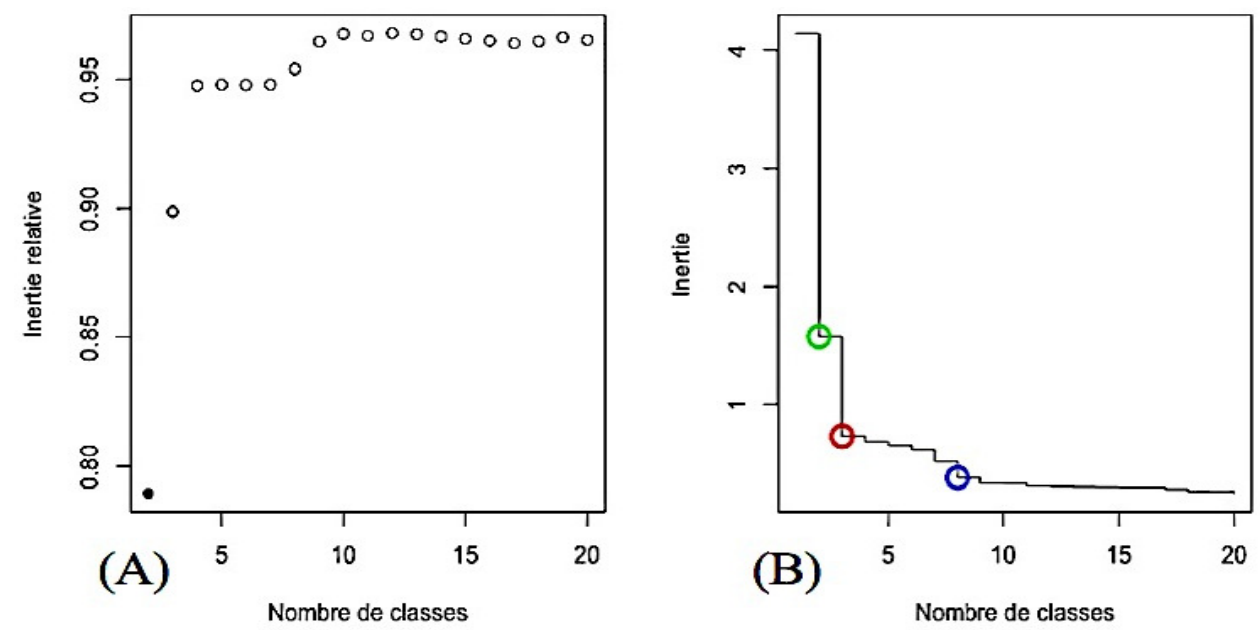

Figure 4 : Perte relative d'inertie (A) et sauts d'inertie des dendrogrammes (B) selon le nombre de classes

Avec le découpage en deux classes (premier niveau de classification) d'un effectif total composé de trois biotypes, l'inertie intragroupe est maximale (faible homogénéité intragroupe) et l'inertie intergroupe minimale (faible hétérogénéité intergroupe), minimisant ainsi le critère d'homogénéité de chaque classe définie, donc la quantité d'informations que révèle la $\mathrm{CAH}$. Cependant, afin de réaliser une analyse beaucoup plus fine, un nombre de classes plus élevé serait plus pertinent. Pour cette raison, le découpage en trois classes est retenu. Trois sauts d'inertie sont notés à 2, 3 et 8 classes (Figure $4 A)$ représentés sur le graphique en vert, en rouge et en bleu respectivement, et justifient ainsi les trois classes hiérarchiques retenues. Le dendrogramme a révélé trois groupes morphologiques (Figure 5). Le premier (en rouge) regroupe $30 \%$ de l'effectif total, le deuxième (en bleu) rassemble les $62 \%$ et le troisième (en noir) réunit les $8 \%$.

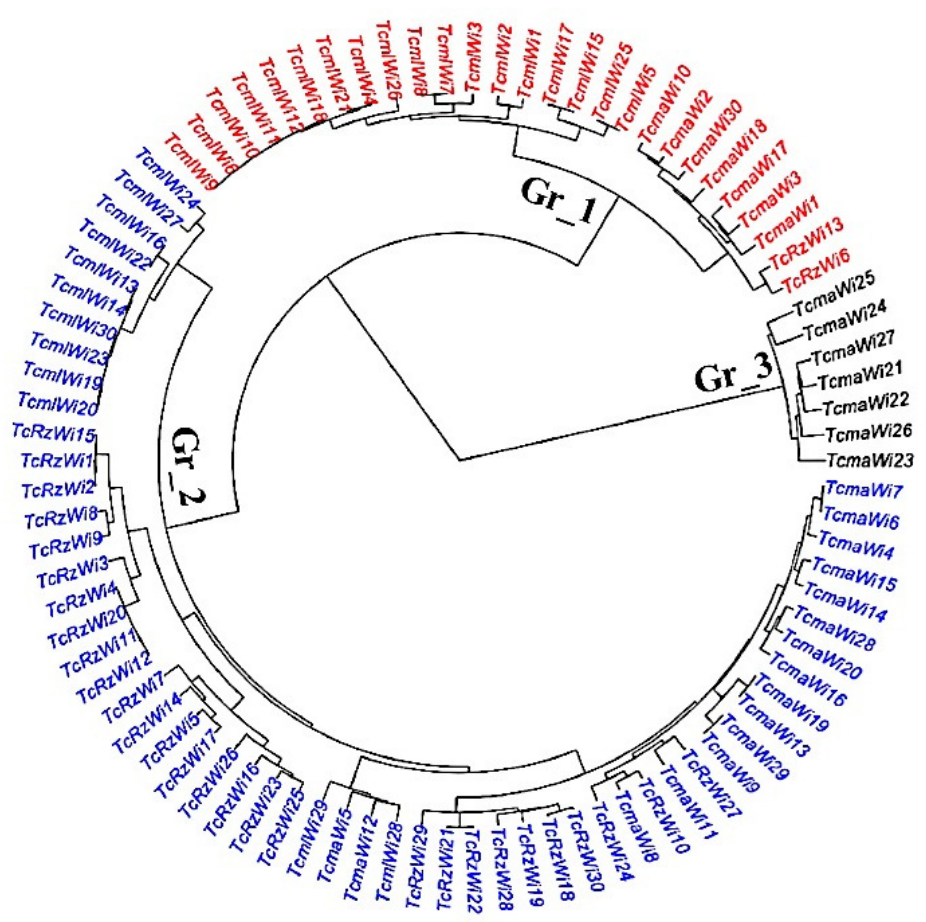

Figure 5 : Dendrogramme de la $\mathrm{CAH}$ 

Herbst (Coleoptera, Tenebrionidae) inféodées à trois céréales à Widou Thiengoli

Seul le biotype maïs compose le groupe_3 (100\%). Les groupe_1 et 2, morphologies identifiées chez les trois biotypes, sont composés en majorité d'individus du biotype mil (67\%) et du biotype riz (50\%) respectivement (Table 4). Le biotype maïs, bien qu'une partie de ses individus (23\%) constitue spécifiquement le groupe_3, sa morphologie est beaucoup plus expliquée par celle du groupe_2 (54\% de ces individus). Les 23\% restants des individus de ce biotype ont la morphologie du groupe_1. $\mathrm{Ce}$ dernier explique la morphologie de la plupart des individus du biotype mil ( $60 \%$ de ses individus). Les individus restants de ce biotype (40\%) ont la morphologie du groupe_2. La quasi-totalité des individus du biotype riz $(93 \%)$ ont la morphologie du groupe_2. Certains des individus de ce biotype $(7 \%)$ trouvent leur morphologie dans le groupe_1 (Table 4).

Tableau 4 : Composition des groupes morphologiques selon l'appartenance des individus qui les composent et répartition des individus de chaque biotype dans les groupes définis

\begin{tabular}{|c|c|c|c|c|c|c|c|}
\hline \multicolumn{4}{|c|}{ Composition des groupes morphologiques } & \multicolumn{4}{|c|}{ Répartition des biotypes dans les groupes } \\
\hline $\begin{array}{ll} & \text { Biotypes } \\
\text { Groupes } & \end{array}$ & Mil & Maïs & Riz & $\begin{array}{ll}\text { Biotypes } & \text { Groupes }\end{array}$ & Gr_1 & Gr_2 & Gr_3 \\
\hline Gr_1 (30\%) & 67 & 26 & 7 & Mil & 60 & 40 & --- \\
\hline Gr_2 (62\%) & 21 & 29 & 50 & Maïs & 23 & 54 & 23 \\
\hline Gr_3 (08\%) & --- & 100 & --- & Riz & 7 & 93 & --- \\
\hline
\end{tabular}

Le groupe_3 a l'inertie intragroupe la plus grande (0.0339). Ce qui révèle l'hétérogénéité morphologique de ce groupe par rapport aux autres, bien qu'il soit composé spécifiquement d'individus du biotype maïs. Les groupes 1 et 2 , constitués en majorité d'individus du biotype mil et $\mathrm{du}$ biotype riz respectivement, ont les variances intragroupes les plus petites $(0.0185$ pour le groupe_ 1 et 0.0262 pour le groupe_2) en particulier le groupe_1 qui est morphologiquement le plus homogène. Les plus grandes inerties intergroupes (8.347) sont notées d'une part entre le groupe_2 dominé par des individus du biotype riz et le groupe_3 constitué uniquement d'individus du biotype maïs et d'autre part entre le groupe_2 et le groupe_1, composé en majorité d'individus du biotype mil. Les groupes_1 et 2 sont morphologiquement les plus proches (Tableau 5).

Tableau 5 : Matrice de dissimilarité selon la distance de Ward entre les groupes morphologiques

\begin{tabular}{c|c|c|c}
\hline Groupes & Gr_1 & Gr_2 & Gr_3 \\
\hline Gr_1 & & 1.239 & 7.521 \\
\hline Gr_2 & 1.239 & & 8.347 \\
\hline Gr_3 & 7.521 & 8.347 & \\
\hline
\end{tabular}

Indice de silhouette: Bien qu'il soit le plus morphologiquement hétérogène, les individus $d u$ groupe_1 sont les mieux regroupés avec le plus grand indice de Silhouette (cluster noir: IS $=0,76$ ) suivis de ceux du groupe_1 (cluster rouge : IS = 0.43) (Figure 6). Ayant des indices de Silhouette négatifs (IS $=-0.018)$, quatre individus du groupe_2 (cluster bleu : IS $=0.30$ ) ne sont pas bien regroupés. II s'agit des individus du biotype mil tels que TcmIWi19, TcmIWi20, TcmIWi23 et TcmIWi30. Après reclassement, ces individus appartiennent au groupe_1 plutôt qu'au groupe_2. 


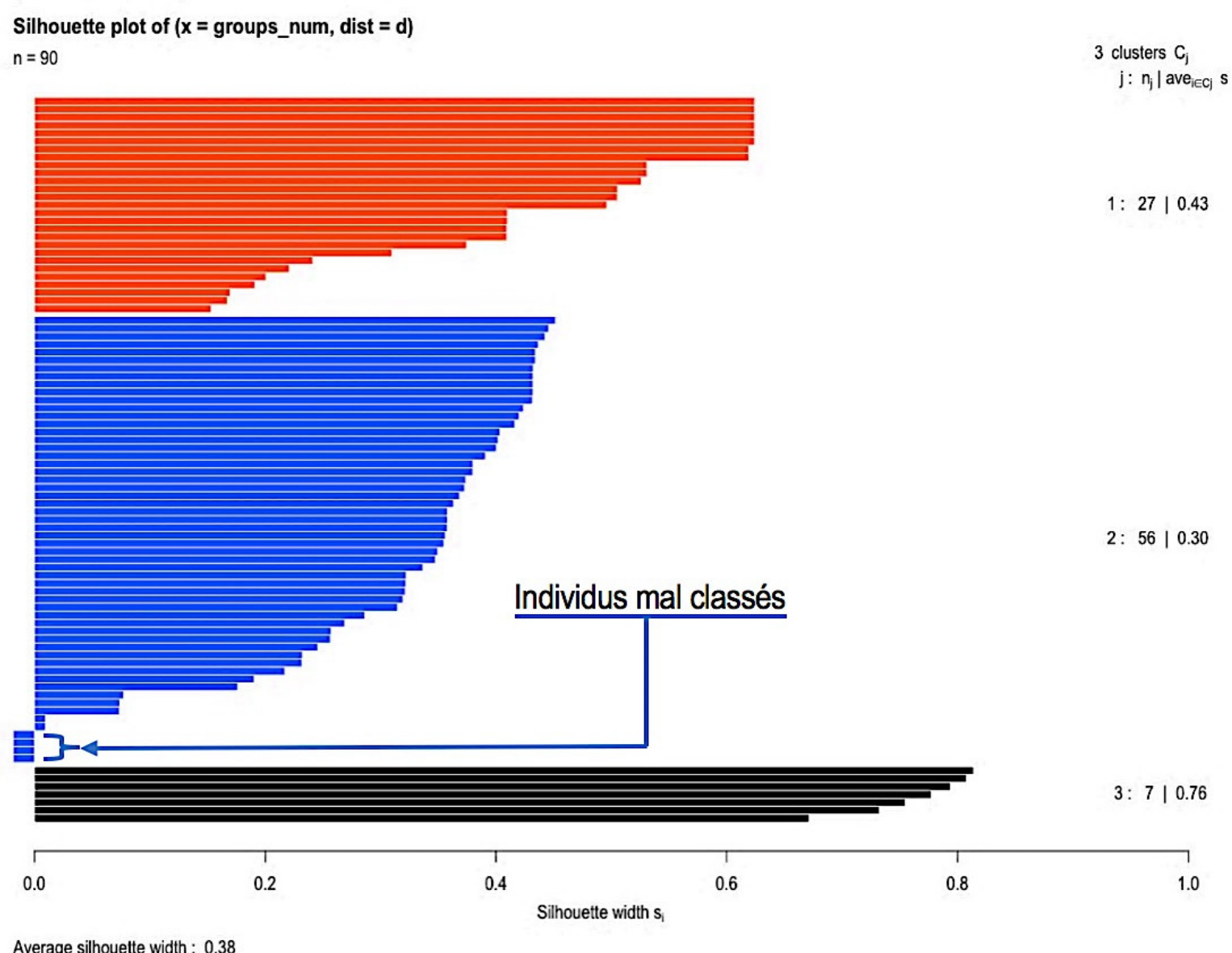

Average silhouette width : 0.38

Figure 6 : Diagramme de Silhouette

\section{DISCUSSION}

Les données brutes ont révélé un effet taille des individus de $T$. castaneum de Widou Thiengoli avec une plus grande taille pour les individus échantillonnés sur du mil par rapport à ceux qui ont émergé sur du maïs et sur du riz. Selon Ming \& Cheng (2012), la performance de développement d'un $T$. castaneum mâle est fortement influencée par la qualité nutritionnelle. La plante hôte serait donc à l'origine de cette différence de taille d'autant plus que selon la FAO (1995), le mil est plus nutritif que le riz et le maïs (Hulse et al., 1980). Néanmoins, les dimensions de la largeur maximale des élytres (Ime), de la largeur du fémur_3 $\left(\mathrm{If}_{3}\right)$ et de la distance entre les yeux ventralement et dorsalement (Dyv et Dyd) restent constantes chez tous les individus de tous les biotypes. Selon Delobel \& Tran (1993) la distance entre les yeux ventralement $(D y v)$ est une clé d'identification de $T$. castaneum de son espèce jumelle Tribolium confusum. La largeur maximale des élytres (Ime), la largeur du fémur_3 $\left(\mathrm{If}_{3}\right)$ et la distance entre les yeux dorsalement
(Dyd) pourraient être aussi spécifiques à l'espèce $T$. castaneum. Avec les données brutes, la grande taille des individus du biotype mil par rapport à ceux des autres biotypes est révélée par la première dimension de l'AD (Dim_1) qui conserve la plus grande part de l'inertie totale. La taille du grain de maïs (poids moyen : $7.8 \pm$ $0.04 \mathrm{~g}$ ) étant plus grande que celle du grain de mil (poids moyen : $0.11 \pm 0.015 \mathrm{~g}$ ), toutes les $C P$ retenues sauf $D p t$ ont des mensurations brutes plus grandes chez les individus du biotype mil par rapport à ceux du biotype maïs. Ainsi, il n'existe pas de rapport direct entre la taille du grain infesté et celle de l'insecte qui l'infeste. Ce même phénomène est observé chez Caryedon serratus sur ses différentes plantes hôtes (Sembene \& Delobel, 1996). C'est donc, dans une première approche, le facteur taille qui distingue le biotype mil des autres. Ce facteur intervient certes dans la différenciation entre biotypes à Widou Thiengoli, mais l'analyse des logvariables transformées montre qu'il est loin d'être le seul. 


\section{Dia et al., J. Appl. Biosci. 2017 Identification morphométrique des populations de Tribolium castaneum}

Herbst (Coleoptera, Tenebrionidae) inféodées à trois céréales à Widou Thiengoli

L'élimination de l'effet taille est expliquée par la baisse de la contribution des variables à la construction des deux dimensions de l'ACP après transformation des données. II s'agit, suivant Dim_1, de la longueur du fémur_3 $\left(L f_{3}\right)$, de la longueur maximale des élytres $(L m e)$, de la longueur du tarse_3 (Lta 3 ), de la longueur du pygidium (Lpy) et de la distance entre le pronotum et le thorax (Dpt)) et, suivant Dim_2, de la largeur du 1er sternite abdominal $\left(I_{1} s\right)$, de la largeur du pronotum $(I p)$ et de la longueur du coxa_3 $\left(L C_{3}\right)$. La longueur du 1er sternite abdominal $\left(L_{1} S\right)$, la longueur du coxa_3 $\left(L C_{3}\right)$ et la largeur du prothorax (IP) suivant Dim_1 et la longueur du tibia_3 $\left(L t i_{3}\right)$ suivant Dim_2 perdent la significativité de leur contribution suite à l'élimination de l'effet taille, permettant ainsi à cette dimension de perdre $8,3 \%$ d'inertie. Par contre la longueur et la largeur maximale de la tête $(\mathrm{Lmt}$, Imt) qui, avant transformation des données, avaient des contributions non significatives (CONT_2 $=5,656$ ), participent significativement à la construction de Dim_2 suite à l'élimination de l'effet taille (Cont_2 $=12,534$ ), permettant ainsi à cette dimension de gagner 3,2\% d'inertie. Suite à la transformation logarithmique des données, l'ACP a montré que, suivant Dim_1, la longueur du tarse_3 (Lta 3 ), la longueur du pygidium (Lpy) et la distance entre le pronotum et le thorax (Dpt)) et, suivant Dim_2, la largeur du 1er sternite abdominal (11s), la largeur du prothorax (IP) et la longueur du coxa_3 $\left(L C_{3}\right)$ restent les CP les plus discriminantes. À taille égale, les barycentres des trois biotypes se rapprochent les uns des autres mais la discrimination du biotype mil par rapport aux autres biotypes reste toujours visible. II ressort de L'AD des log-variables transformées que le biotype mil se différencie des autres par la distance entre le pronotum et le thorax (Dpt), la largeur du pronotum (Ip), la longueur du pygidium ( $L p y)$ et la longueur du tarse et du coxa de la troisième paire de pattes $\left(L t_{3}, L C_{3}\right)$, et les biotypes riz et maïs se différencient au niveau de la largeur du1er sternite abdominal $\left(I_{1} S\right)$ et de la longueur du coxa_3 $\left(L C_{3}\right)$. Le test de significativité des écarts des moyennes de Logtransformées entre biotypes donne la morphologie spécifique à chacun d'entre eux. Ainsi le biotype mil, le plus morphologiquement différent des autres biotypes, possède un abdomen plus long, une $3^{\text {ème }}$ paire de pattes et des élytres plus développés et une plus petite distance entre le pronotum et le thorax. II possède également un pronotum et un 1er sternite abdominal plus large que le biotype maïs. Les individus de T. castaneum du biotype riz ont la particularité morphologique à avoir un coxa de la $3^{\text {ème }}$ paire de pattes plus long par rapport aux autres biotypes et un pygidium plus large par rapport au biotype mil. Le biotype maïs trouve sa particularité morphologique au niveau de son 1 er sternite abdominal plus large par rapport aux autres biotypes et son pygidium plus large par rapport au biotype riz. Les différences morphologiques significatives trouveraient leurs explications dans la composition organoleptique des grains. Le mil est plus riche en protéines, en lipides et en fibres diététiques. Par contre, les grains de riz, sont plus riches en glucoses disponibles et la teneur en lipides des grains de maïs est pratiquement similaire à celle des grains de mil (FAO, 1970). Sachant que la partie périphérique du grain (Germe scutellum, enveloppes et couche aleurone) est plus riche en amidon et en teneurs en protéines, lipides, minéraux et vitamines, elle occupe $40 \%$ du volume du grain de mil contre $17 \%$ et $10 \%$ du volume des grains maïs et riz respectivement (Michel, 1980). Vu la différence des trois céréales dans leur composition organoleptique, le type de substrat alimentaire, étant le support de développement de $T$. castaneum, semble être déterminant dans la différence de formes entre ses biotypes. Trois groupes morphologiques sont révélés par la classification ascendante hiérarchique: le groupe_3, composé uniquement d'individus du biotype maïs, le groupe_1 et le groupe_2, composés chacun d'individus appartenant aux trois biotypes et dominée par ceux des biotypes mil et riz respectivement. Le groupe_3 est le plus morphologiquement hétérogène avec une variance intragroupe la plus forte, mais le mieux regroupé avec un indice de Silhouette le plus grand. Le groupe_1, le plus morphologiquement homogène, possède l'inertie intragroupe la plus faible. Les plus fortes inerties intergroupes sont notées d'une part entre le groupe_3 et le groupe_2 qui sont les plus morphologiquement dissemblables et d'autre part entre le groupe_3 et le groupe_1. Regroupant $62 \%$ de l'effectif total, le groupe_2 a l'indice de Silhouette le plus faible et certains de ses individus qui le composent, sont mal classés dans ce groupe à priori. II s'agit des individus du biotype mil qui, à postériori, se sont retrouvés bien regroupés dans le groupe_1, composé en majorité des individus de ce biotype. Étant donné les grains de mil sont plus favorablesvi au développement de T. castaneum (Gueye et al., 2012), I'homogénéité morphologique des individus intragroupes du biotype mil s'expliquerait par le fait que le mil est la spéculation primaire de $T$. castaneum (Diome et al., 2015) et il y déroulerait tout son cycle de développement tant qu'il soit disponible et les autres céréales ne seraient infestées par $T$. castaneum que lorsque le mil est indisponible ou inaccessible. Sachant que le cycle de développement de $T$. castaneum (Gueye et al., 2012) peut être influencé par la disponibilité de 
nourriture, les adultes, étant de bons voiliers, migrent à partir de stocks infestés à la recherche de nouvelles ressources alimentaires (Delobel \& Tran, 1993), à la suite d'une forte infestation (grains de mil réduits en farine plus ou moins semblable à de la poussière (Pageau, 2006)). Compte tenu des conditions de stockage des céréales dans les magasins de commerce vii à Widou Thiengoli, $T$. castaneum a la possibilité de coloniser des stocks de riz et/ou de maïs suite à l'épuisement des stocks de mil et inversement. Ce qui expliquerait les similarités morphologiques entre certains individus de différents biotypes. Sachant que le maïs a une quantité nutritionnelle meilleure que celle du riz et du mil, les individus du groupe_3 auraient déroulé exclusivement tout leur cycle de développement sur du maïs grâce à sa disponibilité permanente dans les magasins de commerce. La forte inertie intragroupe du groupe_3 révèle une hétérogénéité morphologique des individus du biotype maïs qui composent ce groupe et monterait ainsi leur instabilité morphologique. Bien qu'elle soit réalisée dans une situation géographique (uniquement dans un seul site géographique : Widou thiengoli) qui ne permet pas de confirmer l'existence d'une structuration morphologique de $T$. castaneum selon le support de développement, cette étude a montré des différences de

\section{CONCLUSION}

Les résultats obtenus au cours de cette étude révèlent trois groupes morphologiques que sont le groupe_3 spécifique au biotype maïs, le groupe_2 regroupant la majorité des individus du biotype riz et une partie des individus d'autres biotypes et le groupe_1 fortement représenté par le biotype mil et renferme quelques individus des autres biotypes. La morphologie du groupe_2 est la plus partagée et est reproduite par plus de la moitié des individus de l'effectif total. Le groupes_3, spécifique au biotype maïs, est caractérisée par une morphologie hétérogène entre les individus qui le composent et se révèle être le groupe morphologique le plus dissemblable des autres. Le groupe_1 présente plus d'homogénéité morphologique des individus qui le composent. Outre que les individus du biotype mil qui taille et de forme entre les trois biotypes de cet insecte. L'étude de Diome (2014) qui avait montré que le poids moyen des individus de T. castaneum ayant accompli leur cycle développement sur du mil est légèrement plus élevé que ceux qui l'ont accompli sur du maïs, corrobore la thèse avancée par cette étude sur la différence de taille qui existe entre le biotype mil et les autres. Étant donné que la diversité alimentaire agit sur la diversité morphologique de la bruche de l'arachide (Caryedon serratus) selon les travaux de Sembene \& Delobel (1996) et de la bruche de Niébé (Bruchidius atrolineatus) d'après les travaux de Moumouni et al. (2014), les conditions de stockage des céréales dans les magasins de commerce à Widou Thiengoli qui sont favorables à la diversité génétique de $T$. castaneum (Dia et al., 2014), offrent à ses individus la possibilité d'infester diverses céréales stockées dans la même infrastructure de stockage selon la disponibilité alimentaire. Les différences de formes entre les trois biotypes à Widou Thiengoli révélées par cette étude, ne permettent pas de confirmer une structuration morphologique de $T$. castaneum selon le support alimentaire, mais permettrait de vérifier une éventuelle adaptation morphologique de T. castaneum sur différentes plantes hôtes dans plusieurs sites géographiques.

sont mal regroupés à priori dans le groupe_2 et qui appartiennent au groupe_1 à postériori, tous les individus sont bien classés. La grande taille des individus du biotype mil par rapport aux autres et les différences de formes entre les trois biotypes ressortent de cette étude. La plante hôte s'est montrée être un facteur déterminant dans la discrimination morphologique des populations de cet insecte dans la zone de la grande muraille verte. Cette étude ne permet pas de confirmer une éventuelle adaptation morphologique de $T$ castaneum sur divers supports alimentaires. Cependant une étude morphologique des biotypes de $T$. castaneum dans plusieurs sites géographiques apportent plus de précisions sur l'adaptabilité morphologique de $T$. castaneum à infester différentes céréales entreposées.

REMERCIEMENTS : Ce travail a été financé par OHMi "Tessékéré" Observatoire Homme Milieu sous l'appui financier du LabEx DRIIHM, et le WAPP "World Bank Program" National centres of Excellence. 
Dia et al., J. Appl. Biosci. 2017 Identification morphométrique des populations de Tribolium castaneum Herbst (Coleoptera, Tenebrionidae) inféodées à trois céréales à Widou Thiengoli

\section{REFERENCES BIBIOGRAPHIQUES}

Bloomfield V.A. 2014. Using R for numerical Analysis in Science and Engineering. Chapman \& Hall/CRC The R Series, 359p (ISBN 978-14398844).

Bretaudeau A. (2010). Commerce Transfrontalier et Sécurité Alimentaire en Afrique de l'Ouest : Cas du Bassin Ouest : Gambie, Guinée-Bissau, Guinée, Mali, Mauritanie, Sénégal. 89p

Cornuet J-M, Piry S, Luikart G., Estoup A., and Solignac M. 1999. New Methods Employing Multilocus Genotypes to Select or Exclude Populations as Origins of Individuals. Genetics Society of America, 153: 1989-2000.

Darrroch, JN \& Mosimann, JE. 1985. Canonical and principal components of shape. Biometrika. 72 (2) : $241-252$

Delobel A. et Tran M. (1993). Les Coléoptères des denrées alimentaires entreposées dans les régions chaudes. OSTROM/ CTA, Faune tropicale XXXII. 439p

Dia C. A. K. M., Diome T., Thiaw C., Diop M. et Sembene M. (2014). Impact of storage infrastructures and agroecological areas in genetic demographic evolution of Tribolium castaneum Herbst (Coleoptera: Tenebrionidae) Senegalese populations. International Journal of Science and Advanced Technology (ISSN 2221-8386) Volume 4, Page : 12 - 23.

Diome T. (2014). Biodémographie et diversité génétique des populations de Tribolium castaneum Herbst (Coleoptera, Tenebrionidae) ravageur des grains de mil (Pennisetum glaucum Brown) en stock au Sénégal. Thèse de Doctorat unique en Génétique des populations, Université Cgeikh Anta DIOP de Dakar.

FAO (1970). Table de composition des aliments à l'usage de l'Afrique. FAO, Rome.

FAO (1995). Le sorgho et les mails dans la nutrition humaine, ROME

FAO (1996). Annuaire production: 1995. Rome, Italie, $\mathrm{FAO}, 235 \mathrm{p}$.

Gueye MT., Dogo S., Wathelet JP., Lognay Georges (2011). Lutte contre les ravageurs des stocks de céréales et de légumineuses au Sénégal et en Afrique occidentale : synthèse bibliographique.Biotechnol. Agron. Soc. Environ. 2011 15(1), 183-194.

Guèye, A.C., Diome, T., Thiaw, C., Ndong, A., Guèye, A.N. \& Sembène, M. (2012). Capacity of biodemographic development of Tribolium castaneum Herbst (Coleoptera, Tenebrionidae) and Sitophilus zeamais Motschulsky (Coleoptera, Curculionidae) in stored cereals in Senegal. South Asian Journal of Experimental Biology, 2 (3) : 108-117.

Hulse, J.H., Laing, E.M. \& Pearson, O.E. 1980. Sorghum and the millets : their composition and nutritive value. New York, Academic Press. $997 \mathrm{p}$.

Michel JC(1980).Utilisation potentielle du sorgho dans un systeme intégré de mouture et de pastification. In : l'amélioration des systèmes post-récoltes en Afrique de l'Ouest. Agence de coopération culturelle et technique, Paris.

Ming QL, Cheng C (2012) Influence of nutrition on male development and reproduction in Tribolium castaneum. J Econ Entomol 105:1471-1476

Moumouni D. A., Doumma A., Toufique B. M., Sembene M. (2014). Influence des trois variétés du niébé sur la biologie et la morphométrie de Bruchidius atrolineatus, ravageurs du niébé au Niger. AFPP - Dixième conférence internationale sur les ravageurs en agriculture. MONTPELLIER - 22 ET 23 octobre 2014.

Ouédraogo S. J., W. Théodore Kaboré W. T. et Loada M. (2014). Étude prospective sur les pertes post récoltes en Afrique de l'ouest : cas de quelques pays côtiers et du sahel. Rapport définitif, Institut du Sahel (CILSS). 44p.

Pageau G. (2006). Les Triboliums, vous connaissez ? (2e partie). Ministère de l'environnement. $4 p$

Poisson C., Marnotte P., Ould-Didi H. (1994). Le riz mauritanien en forte progression. Agriculture et développement $n^{\circ} 2.9 p$.

Santos F. (2015). Analyse en Composantes Principales (ACP) : Travaux Pratiques avec le logiciel R. CNRS, UMR 5199 PACEA.

Sembene M. (2000). Variabilité de l'Espaceur Interne Transcrit (ITSI) de I'ADN ribosomique et polymorphisme des locus microsatellites chez la bruche Caryedon serratus (Olivier) : différenciation en races d'hôtes et infestation de l'arachide au Sénégal. THESE DE DOCTORAT D'ETAT ES SCIENCES, Université Cheikh Anta DIOP de Dakar.

Sembene M. et Delobel A. (1996). Identification morphométrique de populations soudanosahéliennes de bruche de l'arachide, Caryedon serratus (Olivier) (Coleoptera, Bruchidae). Journal of African Zoology 110 (5). 10p.

Sezonlin M. (2006). Phylogeographie et genetique des populations du foreur de tiges de céréales 
Busse olafusca (fuller) (lepidoptera, noctuidae) en Afrique subsaharienne, implications pour la lutte biologique contre cet insect. Thèse de doctorat de l'université de Paris XI-Orsay. 152p.

\footnotetext{
i Selon le critère de Coude, une variable est considérée comme $\mathrm{CP}$ que lorsqu'elle donne un sens à l'axe ou la dimension considérée. La contribution de la CP est toujours significative c'est-à-dire supérieure à la contribution moyenne (Fmoy =1/n, avec $n=$ nombre de variables utilisées pour réaliser les ACP). ii Est considéré, dans cette étude, comme biotype, un groupe d'individus qui ont émergé de la même céréale. Les trois groupes d'individus qui émergent chacun sur une céréale donnée (mil, maïs et riz) constituent les trois biotypes.

iii Population totale structurée ou non en deux ou plusieurs groupes.

iv Plus une variable est proche d'une dimension (axe) principale de l'ACP, plus elle est liée à elle.

$\checkmark$ Variables qui contribuent significativement à la construction d'une dimension suite à l'élimination de l'effet taille.

vi La dare moyenne de développement de $T$. castaneum est plus courte sur les grains de mil $(29,38 \pm 1,45$ jours) que sur ceux du maïs (36,3 $\pm 1,42$ jours) (Gueye et al., 2012).

vii Le mil, le riz, le maïs, sont stockés en permanence dans ces magasins.
} 